\title{
A Case of Odontogenic Fistula Misdiagnosed as Cutaneous Ulcer on the Alar-Facial Angle
}

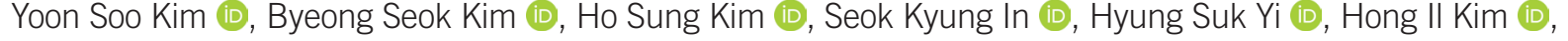 \\ Jin Hyung Park (D), Hyo Young Kim (D) \\ Department of Plastic and Reconstructive Surgery, Kosin University College of Medicine, Busan, Korea
}

\begin{abstract}
Odontogenic cutaneous fistula of dental origin at the alar-facial groove is rarely reported. As many patients present with only skin lesions without dental problems, it is often confused with a cyst, abscess or pyogenic granuloma. We report the case of a 52-year-old man who presented with a relapsing cutaneous ulcer in the left alar-facial groove area. Ultrasonography findings suggested a ruptured epidermal cyst, and an excisional biopsy was performed. At 10 days after the surgery, wound dehiscence and pus discharge were observed. Computed tomography revealed a sinus tract, and a diagnosis of odontogenic cutaneous fistula was made. After consultation with the dental department, endodontic treatment was performed. During reoperation, a remnant fistula from the left alar-facial groove area to the left upper central incisor was observed, for which fistulectomy was performed. The patient remained disease-free postoperatively. With early accurate diagnosis, patients with odontogenic cutaneous fistula can be protected from unnecessary surgical intervention and ineffective antibiotic therapy.
\end{abstract}

Keywords: Cutaneous fistula; Oroantral fistula; Epidermal cyst

\section{Introduction}

Odontogenic cutaneous fistula is characterized by the presence of a draining sinus fistula originating from a localized area with pus and surrounded by granulation tissue in the alveolar bone of the affected tooth.

It is frequently misdiagnosed as a cyst, abscess, pyogenic granuloma, and so on, as many patients visit clinics because of skin problems and without experiencing toothaches [1]. Accurate diagnosis is most important for odontogenic cutaneous fistula because the lesion is often misdiagnosed as being of non-odontogenic origin, which results in inappropriate management such as a prolonged course of antibiotics and repeated surgical treatment. It is usually located around the mandibular body, chin, buccal cheek, or submandibular region [2,3].

We report the case of a 52-year-old man who presented with a relapsing cutaneous ulcer due to odontogenic cutaneous fistula in the alar-facial groove, a site rarely reported without any dental symptoms. The study was performed in accordance with the principles of the Declaration of Helsinki. The patient provided written informed consent for the publication and the use of his images.

\section{Case}

A 52-year-old man presented with a relapsing cutaneous ulcer on the left alar-facial groove area (Fig. 1). The patient had received antibiotics treatment at a local clinic

\section{Case Report}

Received: August 21, 2020

Revised: September 28, 2020

Accepted: September 29, 2020

\section{Corresponding author:}

Hyo Young Kim, M.D.

Department of Plastic and Reconstructive Surgery, Kosin University College of Medicine, 262 Gamcheon-ro, Seo-gu, Busan 49267 Korea

Tel: +82-51-990-6131

Fax: +82-51-990-6312

E-mail: hose3290@naver.com

This article was presented as an E-poster at the 77th Congress of the Korean Society of Plastic and Reconstructive Surgeons on November 8-10, 2019, in Seoul, Korea.

This is an Open Access article distributed under the terms of the Creative Commons Attribution Non-Commercial License (https://creativecommons.org/licenses/by-nc/4.0/) which permits unrestricted non-commercial use, distribution, and reproduction in any medium, provided the original work is properly cited.

(c) 2020 Korean Wound Management Society 
about three times several years ago after being diagnosed with an epidermal cyst in the same area. The patient had no history of dental procedures. On physical examination at the time of the patient's first visit to our institution, an ulcerative lesion of $0.3 \times 0.5 \mathrm{~cm}$ in size was observable with no tenderness in the left alar-facial groove area.

Excisional biopsy was performed in accordance with the ultrasonography findings of a suspected ruptured epidermal cyst in the area (Fig. 2). Pathological findings of the lesion biopsy indicated chronic inflammation with fibrosis. Ten days after the surgery, wound dehiscence was found with a pus-like discharge. A computed tomography (CT) scan was performed and revealed a fistula involving the tooth root in the surgical area (Fig. 3).

On the basis of the patient's clinical history and radiological findings, a comprehensive diagnosis of odontogenic cutane-

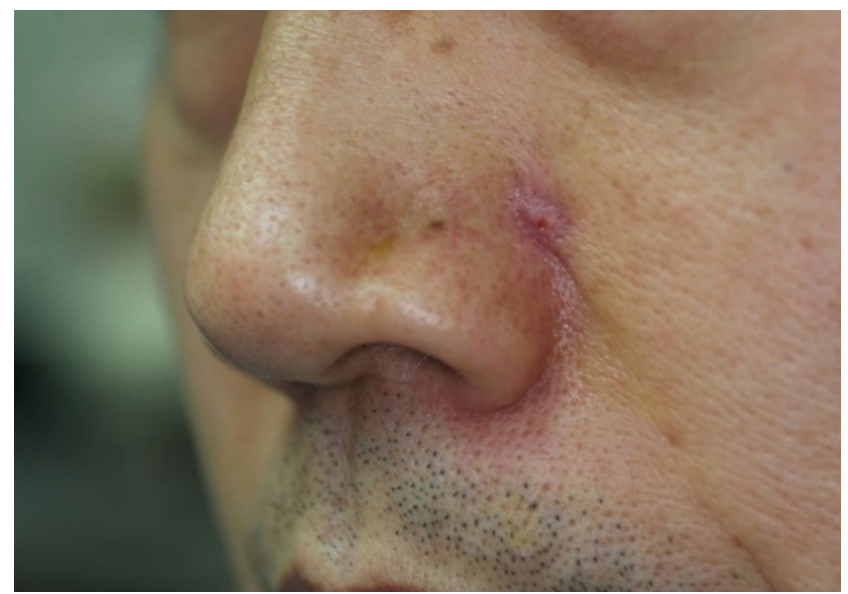

Fig. 1. Preoperative clinical photograph showing a cutaneous ulcer. A 52-year-old man with a cutaneous ulcer on the left alar-facial groove area.

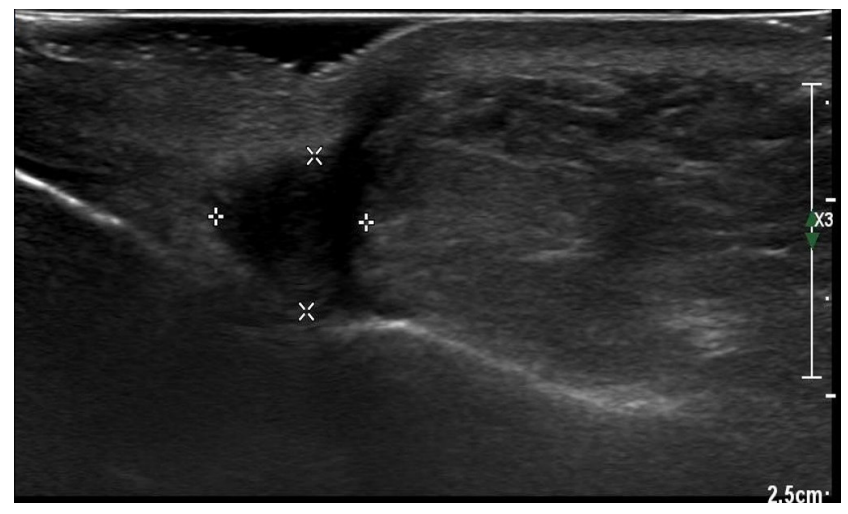

Fig. 2. Ultrasonographic image of the suspected ruptured epidermal cyst. ous fistula was made. After consultation with the dental department, endodontic treatment was performed for the chronic simplex periodontitis of the left upper canine. A week later, the patient was operated on again under local anesthesia, and a remnant fistula progressing into the deeper region was observed in the surgical area. After injection of Gentian violet,

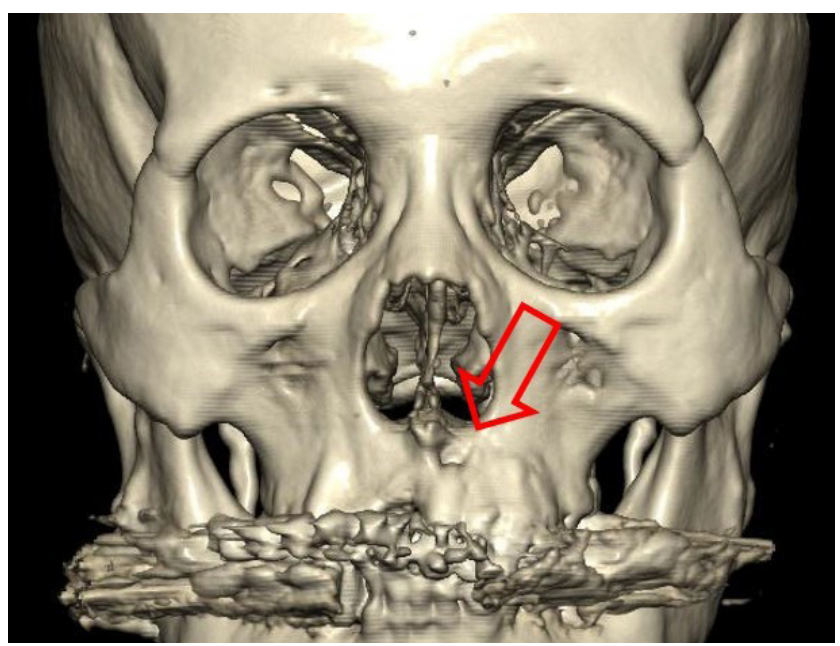

Fig. 3. The three-dimensional reconstruction of computed tomography image. The image shows a focal bony cortical defect at the left upper incisor root and a sinus tract between the left alar-facial groove area skin and beneath the left upper central incisor tooth. The arrow indicates the fistula tract.

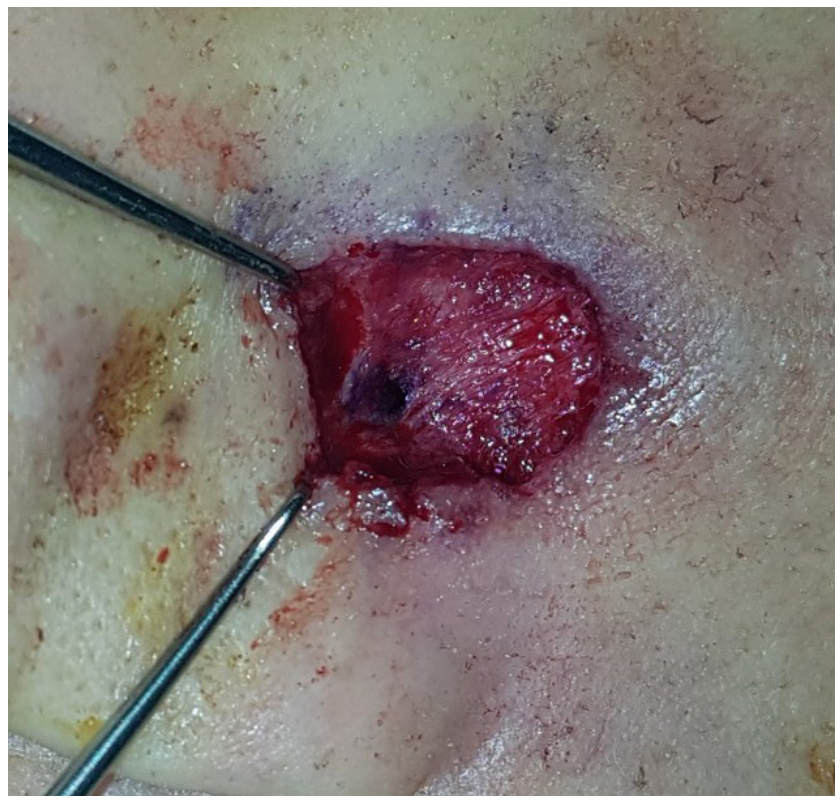

Fig. 4. Intraoperative photo showing an odontogenic cutaneous fistula. Odontogenic cutaneous fistula of the left alar-facial groove area. Intraoperative photograph with Gentian violet staining showing a remnant fistula connected to the incisor root. 


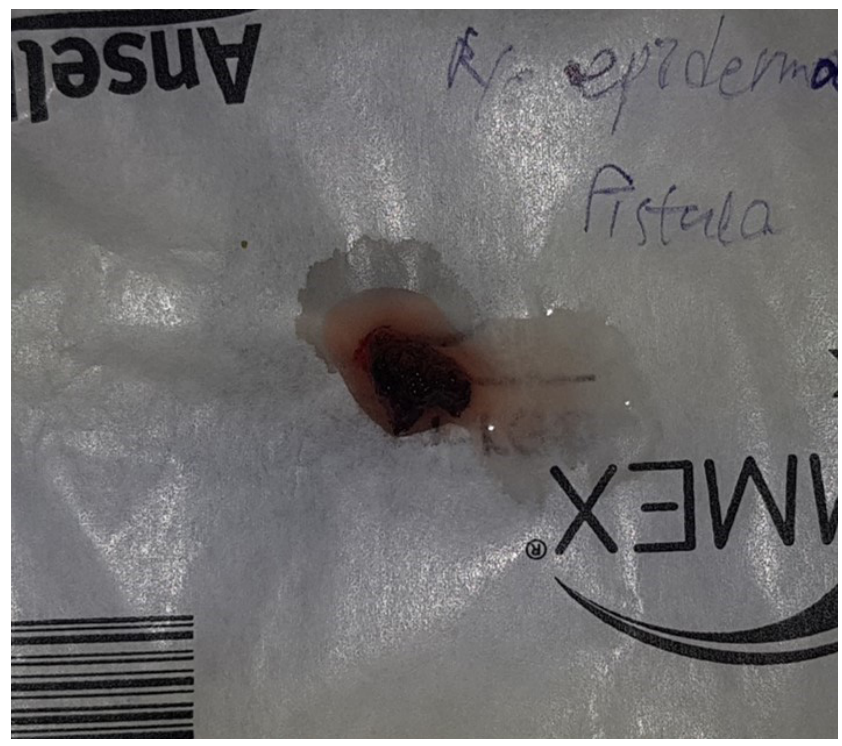

Fig. 5. Resected fistula tract. Odontogenic cutaneous fistula of the left alar-facial groove area.

a fistula from the left alar-facial groove area to the left upper central incisor was observed (Fig. 4), and fistulectomy was performed (Fig. 5). No recurrence was found postoperatively (Fig. 6).

\section{Discussion}

The present case was diagnosed as odontogenic cutaneous fistula on the basis of the CT scan obtained during the patient's visit for a recurring cutaneous ulcer in the alar-facial groove area. It was well cured with dental treatment and fistulectomy.

Odontogenic fistula refers to the progression of inflammation, caused by dental caries or trauma, to pulpal degeneration and pus release into the gums and surrounding tissues. If the apical periodontitis worsens, continuous or intermittent draining can occur through openings by forming a sinus tract into, for example, alveolar bone, skin, or natural cavities such as the maxillary sinus. Cutaneous sinus fistula occurs when purulent products of dental pulp infection and necrosis spread along the path of least resistance from the root apex to the skin on the face. Such infections are caused by dental caries, fractures, and root canal or dental prosthetic treatment, and if the pus drains through a sinus tract into the skin, it often forms an erythematous nodule without tenderness [4].

A cutaneous fistula formed by a dental abscess typically appears as an erythematous nodule that periodically releases pus from the facial skin; tooth related symptoms occur only in

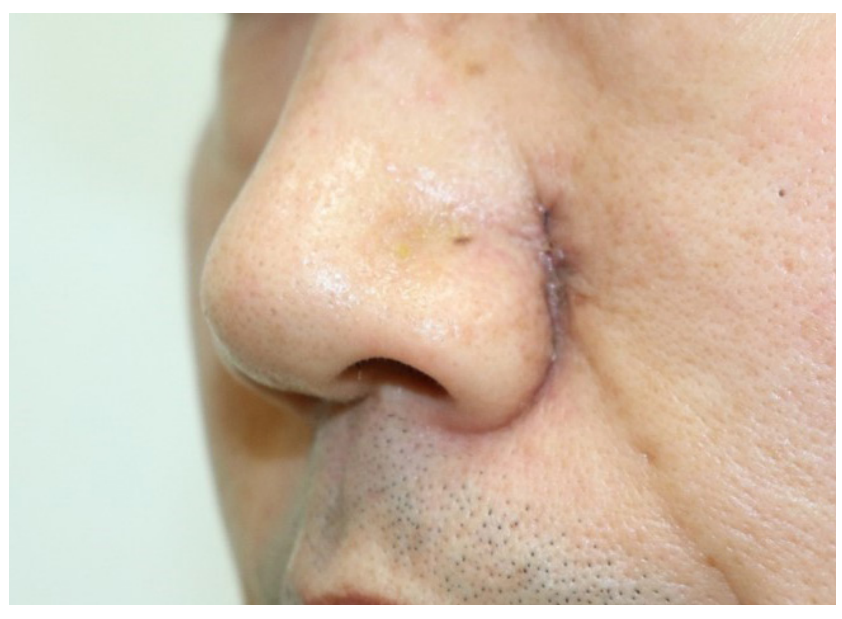

Fig. 6. Two-week postoperative photo. No recurrence was found at 2-week postoperative clinical photograph.

$50 \%$ of patients [5]. In patients with dental pain or symptoms, the cause and appropriate dental treatment may be easy to identify, but the disease can easily be overlooked if patients visit other clinics with an erythematous nodule that does not display sustained symptoms, as demonstrated in this case. The reason for the high rate of asymptomatic dental abscesses is the characteristic of weakly progressing tooth infection, and because the periodic drainage through the tract prevents pain, swelling, or pressure.

These tracts tend to occur more frequently from infection in the mandibular teeth $(80 \%)$ than in the maxillary teeth $(20 \%)$ [6]. This tendency is believed to be the result of the effects of anatomical structures and gravity. In the case of a maxillary tooth periapical abscess, the pus is highly likely to escape into the oral vestibular space owing to gravity. However, in the mandible, as the direction is opposite to gravity, pus is not easily exported to the oral vestibular space, instead melting cancellous bone and creating a tract into the cortical bone, which results in a fistula to the skin. As a result, many cases seem to be of mandibular origin. In $50 \%$ of cases of fistula of mandibular origin, pus is leaked from the mandibular or canine teeth. Most odontogenic cutaneous fistulas were located on the mandibular body or chin, originating from a mandibular tooth. According to the results of a study of fistula locations, if the formation of the cutaneous fistula is toward the alar-facial groove area as in the present case, it is generally caused by inflammation and pus in the canine or incisor, and diagnosis of the dental abscess origin is more difficult [5].

The traditional consensus on fistulas in general is that their formation does not exhibit a sexual predilection, with no sig- 
nificant relationship between prevalence and age either. However, cutaneous sinus tract of dental abscess origin is more common in males than in females, with a higher incidence and duration in patients aged $>60$ years [4].

Palpating the tissue around the cutaneous fistula can cause secretions such as purulent discharge to flow out. However, as patients rarely complain of dental symptoms, both a physical examination of the teeth in the oral cavity (which in many cases show inconclusive findings) and dental radiography is essential for diagnosis [1]. Histological examination also usually reveal nonspecific findings such as chronic inflammatory cells, granulation tissue, and pseudoepitheliomatous hyperplasia, making diagnosis difficult. On radiological examination such as panorex X-ray, sites of progressive apical periodontitis appear radiolucent. Tooth or fistula inflammation can be detected using $\mathrm{CT}$, which is known to be more useful than panoramic view radiography [7]. Ultrasonography was chosen as the first imaging examination in this case, which was difficult to diagnose because the fistula was difficult to find, resulting in ruptured epidermal cyst findings [8-10]. Although there are not many studies on the use of ultrasonography for diagnosing odontogenic fistulas, both positive and negative reports do exist in the literature. Further research is needed on the effectiveness of ultrasonography in diagnosing odontogenic cutaneous fistula.

The differential diagnoses reported in previous studies include pyogenic granulomas, furuncles, sebaceous cysts, foreign body granulomas, subcutaneous mycosis, and neoplastic processes such as squamous and basal cell carcinomas [3]. After performing the first excisional operation in this case, histopathological findings corresponded with chronic inflammation with fibrosis. This case was difficult to diagnose accurately through biopsy alone. Therefore, although odontogenic cutaneous fistulas do not commonly occur in the alar-facial groove area, a CT scan is recommended for ulcers with recurring pus secretion in this area. If skin lesions are suspected to be of dental abscess origin, performing a noninvasive radiological examination is appropriate. If the patient does not respond well to continued treatment or if the patient's condition seems to alternate between improvement and exacerbation, a radiological examination is necessary to discriminate odontogenic cutaneous fistula from other conditions. Unnecessary biopsy should be avoided, as it can worsen inflammation and scarring. Delayed accurate diagnosis leads to unnecessary antibiotic treatment and improper surgical intervention, whereas clinical suspicion and timely radiographic examination can help prevent scars and unnecessary wasting of time and mon- ey due to biopsy or incisional drainage [11].

In a study by Rega et al. [12], aerobes were cultured from odontogenic infections at a higher rate than anaerobes, with more Gram-positive cocci than Gram-negative rods [12]. In this case, Gram-positive Staphylococcus epidermidis was identified. However, since chronic simplex periodontitis of the left upper canine was diagnosed after dental consultation, and endodontic treatment followed by fistulectomy led to successful treatment with no recurrence, the fistula was presumed to have originated from a dental root with a staphylococcal infection rather than from normal skin flora.

The obvious treatment for odontogenic cutaneous fistula is root canal treatment for recoverable teeth and removal of the source of infection by extracting teeth that are unrecoverable. The most effective empirical antibiotics to administer as a first-line treatment are clindamycin or a combination of penicillin and metronidazole [13].

Few cases of odontogenic cutaneous fistulas, especially those occurring in the alar-facial groove area as in the present case, have been reported in domestic plastic surgery literature and may be unfamiliar to clinicians. As in this case, odontogenic cutaneous fistula should be considered if ulcerative lesions and abscesses on the face accompanied by chronic inflammation do not respond well to antibiotics or repeated drainage treatments.

\section{Conflict of interest}

No potential conflict of interest relevant to this article was reported.

\section{ORCID iDs}

Yoon Soo Kim

Byeong Seok Kim

Ho Sung Kim

Seok Kyung In

Hyung Suk Yi

Hong Il Kim

Jin Hyung Park

Hyo Young Kim https://orcid.org/0000-0002-8073-5511

https://orcid.org/0000-0003-1375-1092

https://orcid.org/0000-0002-6030-3199

https://orcid.org/0000-0003-4346-7949

https://orcid.org/0000-0002-9584-8201

https://orcid.org/0000-0002-5505-9945

https://orcid.org/0000-0001-9415-2467

https://orcid.org/0000-0002-1834-5766

\section{References}

1. Cohen PR, Eliezri YD. Cutaneous odontogenic sinus simulating a basal cell carcinoma: case report and literature re- 
view. Plast Reconstr Surg 1990;86:123-7.

2. Witherow H, Washan P, Blenkinsopp P. Midline odontogenic infections: a continuing diagnostic problem. $\mathrm{Br} \mathrm{J}$ Plast Surg 2003;56:173-5.

3. Chowdri NA, Sheikh S, Gagloo MA, et al. Clinicopathological profile and surgical results of nonhealing sinuses and fistulous tracts of the head and neck region. J Oral Maxillofac Surg 2009;67:2332-6.

4. Lee EY, Kang JY, Kim KW, et al. Clinical characteristics of odontogenic cutaneous fistulas. Ann Dermatol 2016;28: 417-21.

5. Kim SH, Park SJ, Oh JJ, et al. A case of cutaneous sinus tract of dental origin. Ann Dermatol 2002;14:235-8.

6. Kishore Kumar RV, Devireddy SK, Gali RS, et al. Cutaneous sinuses of cervicofacial region: a clinical study of 200 cases. J Maxillofac Oral Surg 2012;11:411-5.

7. Bodner L, Bar-Ziv J. Cutaneous sinus tract of dental origin: imaging with a dental CT software programme. Br J Oral Maxillofac Surg 1998;36:311-3.
8. Shobatake C, Miyagawa F, Fukumoto T, et al. Usefulness of ultrasonography for rapidly diagnosing cutaneous sinus tracts of dental origin. Eur J Dermatol 2014;24:683-7.

9. Coulier B. Blind submandibular odontogenic fistula: ultrasound and CT findings. Diagn Interv Imaging 2020;101: 505-6.

10. Fernandez-Canga P, Perez-Bustillo A, Rodriguez-Prieto M. Cutaneous lesion of dental origin: convenience of dermatological ultrasound diagnosis by an experienced specialist. Egypt J Dermatol Venerol 2016;36:18.

11. Chang LS. Common pitfall of plastic surgeon for diagnosing cutaneous odontogenic sinus. Arch Craniofac Surg 2018;19:291-5.

12. Rega AJ, Aziz SR, Ziccardi VB. Microbiology and antibiotic sensitivities of head and neck space infections of odontogenic origin. J Oral Maxillofac Surg 2006;64:1377-80.

13. Bhagania M, Youseff W, Mehra P, et al. Treatment of odontogenic infections: an analysis of two antibiotic regimens. J Oral Biol Craniofac Res 2018;8:78-81. 